This item was submitted to Loughborough's Research Repository by the author.

Items in Figshare are protected by copyright, with all rights reserved, unless otherwise indicated.

\title{
Robust stability conditions for remote SISO DMC controller in networked
}

\section{control systems}

PLEASE CITE THE PUBLISHED VERSION

PUBLISHER

(c) Elsevier

VERSION

AM (Accepted Manuscript)

LICENCE

CC BY-NC-ND 4.0

\section{REPOSITORY RECORD}

Huang, Jian, Yongji Wang, Shuang-Hua Yang, and Qi Xu. 2019. "Robust Stability Conditions for Remote SISO DMC Controller in Networked Control Systems". figshare. https://hdl.handle.net/2134/4362. 
This item was submitted to Loughborough's Institutional Repository (https://dspace.lboro.ac.uk/) by the author and is made available under the following Creative Commons Licence conditions.

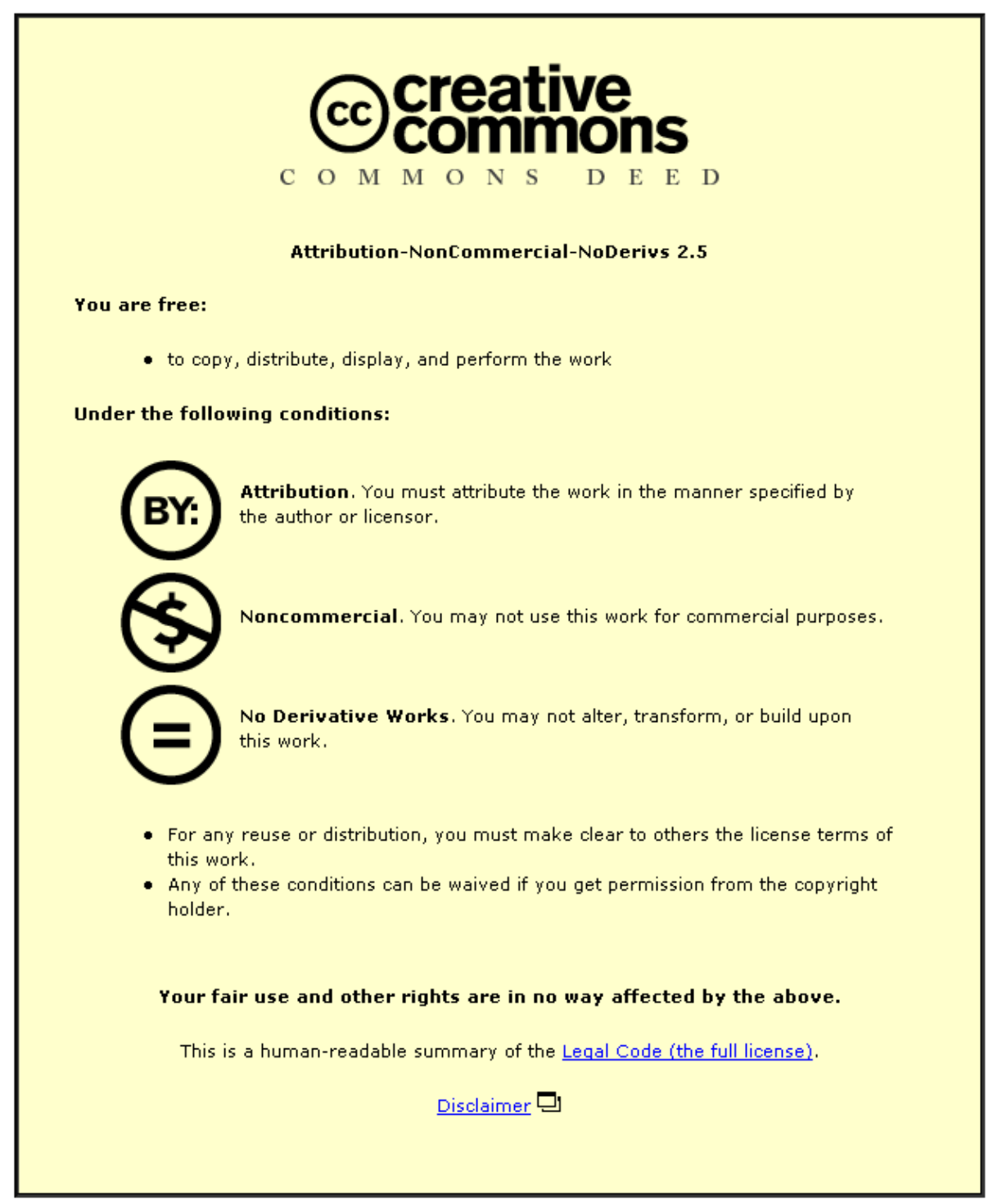

For the full text of this licence, please go to: http://creativecommons.org/licenses/by-nc-nd/2.5/ 


\title{
Robust Stability Conditions for Remote SISO DMC Controller
}

\section{in Networked Control Systems}

\author{
Jian Huang ${ }^{\mathrm{a}, \mathrm{b}, *}$, Yongji Wang ${ }^{\mathrm{a}, \mathrm{b}}$ and Shuang-Hua Yang ${ }^{\mathrm{c}}$ \\ ${ }^{a}$ Department of Control Science \& Engineering, Huazhong University of Science and Technology, 430074, \\ Hubei, Wuhan, China \\ ${ }^{\mathrm{b}}$ Key Lab. for Image Processing \& Intelligent Control of Education Ministry of China, Hubei, Wuhan, China \\ ${ }^{\mathrm{c}}$ Computer Science Department, Loughborough University, Leicestershire LE11 3TU, U.K.
}

\begin{abstract}
A two level hierarchy is employed in the design of Networked Control Systems (NCSs) with bounded random transmission delay. At the lower level a local controller is designed to stabilize the plant. At the higher level a remote controller with the Dynamic Matrix Control (DMC) algorithm is implemented to regulate the desirable set-point for the local controller. The conventional DMC algorithm is not applicable due to the unknown transmission delay in NCSs. To meet the requirements of a networked environment, a new remote DMC controller is proposed in this study. Two methods, maximum delayed output feedback and multi-rate sampling, are used to cope with the delayed feedback sensory data. Under the assumption that the closed-loop local system is described by one FIR model of an FIR model family, the robust stability problem of the remote DMC controller is investigated. Applying Jury's dominant coefficient lemma and some stability results of switching discrete-time systems with multiple delays; several stability criteria are obtained in the form of simple inequalities. Finally, some numerical simulations are given to demonstrate the theoretical results.
\end{abstract}

Keywords: Model predictive control (MPC); Dynamic matrix control (DMC); Stability; Delay; Multi-rate sampling; Networked control systems (NCSs)

\footnotetext{
* Corresponding author. Tel. +86 278754 0014; fax: +86 278754 3130.E-mail: huang_jan@mail.hust.edu.cn
} 


\section{Introduction}

Feedback control systems wherein the control loops are closed through a real-time network are usually called Networked Control Systems (NCSs). The main feature of a NCS is that the components (sensors, controller and actuators) of the system are not connected directly by wires but using a network. In recent times various control units based on real-time networks have been used in a wide variety of applications, but are especially promising when the systems are implemented through the Internet, as benefits can be achieved through retrieval of, and reaction to, data from anywhere around the world at any time.

Although the network makes it convenient to control large distributed systems, there also exists many control issues which cannot be addressed using conventional control theory, including the problems of network delay and data dropout, sampling and transmission methods. To solve these problems many studies have been performed so far. Nilsson [1] analyzed NCSs in the discrete-time domain. He further modeled the network delays as constant, independently random, and random but governed by an underlying Markov chain. Hassibi et al [2] modeled NCSs with data dropouts as Asynchronous Dynamical Systems (ADSs). Branicky et al [3] discussed in detail the problem of how to guarantee the stability of NCSs when various transmission delays or data packet dropouts occur. Zhivoglyadov et al [4] studied a systematic networked control method designed specifically to handle the constraints of the networked realization of a LTI control system. Montestruque [5] dealt with both the state and output feedback control problems in model-based NCSs. Other theories and applications of NCSs can be found in [6-12].

In this study special attention is paid to the model based output feedback control approach proposed in [5]. A typical implementation of this method is illustrated by Fig. 1. In this system construction an estimated process model is held in the remote site to approximate the actual plant behavior when sensory data cannot be 
available due to transmission delay or data package loss. The sampling switch indicates whether the network works normally. If the switch is open, the remote controller does not receive new feedback data and the plant is regulated in an open-loop system. When the network communication is recovered, this switch is closed and the predicted plant output is updated by the available feedback measurements.

Model Predictive Control (MPC), also known as moving horizon control or receding horizon control, is a popular technique for the control of slow dynamical systems, such as those encountered in chemical process control in petrochemical, pulp and paper industries, and in gas pipeline control. Dynamic Matrix Control (DMC) is one of the first commercial implementations of MPC, which was first presented by Cutler et al. in [13]. In the analysis and design of a control system, robustness is always a vitally important issue. In any real, industrial applications there inevitably exist uncertainties concerning unmodeled dynamics, parameter variations, measurement noise, actuator noise, etc. Hence there is a need to study the robust stability of the closed-loop system where model uncertainty is explicitly taken into account. Badgwell introduced a method for deriving robust stability conditions for single-input/single-output (SISO) MPC algorithms, based on an application of Jury's dominant coefficient lemma [14]. The model uncertainty is parameterized by a range of possible plant impulsive responses. Dai extended this method to a new presentation of SISO DMC based on a Finite Impulsive Response (FIR) model [15]. Webber et al. derived a new condition that guarantees robust stability for a set of stable, linear time-invariant plants controlled by using a Simplified Model Predictive Control algorithm (SMPC) [16]. Torgashov considered the problem of determining the robust-stabilizing values of the control (M) and prediction (P) horizons for systems with predictive controllers [17]. Muske and Badgwell present a general disturbance model that accommodates unmeasured disturbances entering through the process input, state, or output [18]. The robust stability problem of the closed-loop system was considered 
as well. In [19], Zhang answered the question: "Does a Model Predictive Controller designed for one model stabilize another model?” In [20], Santos and Biegler addressed another question: “Given an MPC algorithm, how much mismatch can it tolerate and how can we assess this mismatch for stability of the MPC controller?” Whereas, neither the effects of the networked environment nor those of time delay were considered in any of the related studies.

With the development of communication networks, the centralized control structure has gradually been replaced by distributed control in large-scale systems. There is some research on distributed MPC or networked MPC, including Camponogara et. al [21], Dunbar and Murray [22], Li and Zhang [23, 24], and Mercangöza and Doyle [25]. In these studies, much effort has been taken to obtain improved or optimal performance in a distributed networked environment. For instance, Mercangöza and Doyle proposed a DMPC framework that provides significant improvement in control performance over completely decentralized MPC controllers [25]. Li et al. developed an iterative algorithm for distributed MPC based on Nash optimality for large-scale processes [23-24]. Stability problems are either not addressed or only addressed for the nominal system model [22-24]. A novel Networked Predictive Control (NPC) scheme is proposed in [26-27] to overcome the effects of network delay and data dropout, where the stability criteria of closed-loop NPC systems are presented. However, this research is also based on a nominal state-space model and is not the model predictive control study that we are concerned with.

As illustrated above, the robust stability problem of networked MPC has not been fully dealt with so far, which is of importance in the application of MPC in practical control applications. To fill this gap, we study the robust stability of Model Predictive Control systems using feedback data via communication networks.

Because the DMC algorithm is widely accepted in industry, we chose it as the advanced control scheme 
of the remote controller in our two level system structure based on the network, which is illustrated in depth in Section 3.1. This paper is organized as follows: section 2 reviews some preliminary knowledge about the definition of model uncertainty and the newly presented DMC algorithm based on an FIR model. Section 3 gives the main results of this study. First of all, the control system structure based on the network is presented. Two methods are then given using different methods of updating the remote DMC controller. The robust stability results of this remote DMC controller are obtained in both cases. Finally, some numerical simulations are given in section 4 to demonstrate our theoretical results.

\section{Preliminaries}

First, let us review the SISO DMC algorithm based on the FIR model and its fundamental results.

\subsection{FIR Model Family}

A linear, stable, SISO plant with finite gain can be described by an FIR model $h$ with $N$ coefficients [14]:

$$
y(t)=\sum_{i=1}^{N} h(i) u(t-i), t \in \mathbf{Z} .
$$

In this representation, the plant output at time $t$ is denoted by $y(t)$, which depends on $N$ past input values $u(t-i) . \mathbf{Z}$ is the set of integers.

The controller uses a nominal FIR model $\hat{h}$ to predict the future values of plant output at time $t+j$ :

$$
\hat{y}(t+j)=\sum_{i=1}^{N} \hat{h}(i) u(t+j-i)+\hat{d}(t), t \in \mathbf{Z}
$$

where the nominal model $\hat{h}$ is in general different from the actual model $h$. The term $\hat{d}(t)$ is given by

$$
\hat{d}(t)=y(t)-\hat{y}(t), t \in \mathbf{Z}
$$

By adding this term, the predicted plant output is updated all the time by the received feedback data. It is helpful to eliminate the steady bias in the whole closed-loop system. 
The prediction model (2) can be split into a past contribution $\hat{y}_{p}(t+j)$ and a future contribution $\hat{y}_{f}(t+j)$ :

$$
\begin{aligned}
& \hat{y}(t+j)=\hat{y}_{f}(t+j)+\hat{y}_{p}(t+j)+\hat{d}(t), \\
& \hat{y}_{f}(t+j)=\sum_{i=1}^{j} \hat{h}(i) u(t+j-i), \\
& \hat{y}_{p}(t+j)=\sum_{i=j+1}^{N} \hat{h}(i) u(t+j-i) .
\end{aligned}
$$

It is apparent that only the future contribution $\hat{y}_{f}(t+j)$ in Eq. (4) is unknown.

Definition 1 [14]. The family of plants $\pi$ consists of all possible impulsive responses lying between given maximum $\bar{h}$ and minimum $\underline{h}$ limits:

$$
\pi=\{h: \underline{h}(i) \leq h(i) \leq \bar{h}(i), \forall i=1,2, \cdots N\}
$$

Definition 2 [14]. The total mismatch $M$ for a single plant $h$ is defined as

$$
M=\sum_{i=1}^{N}|h(i)-\hat{h}(i)|
$$

Definition 3 [14]. The maximum total mismatch is defined as

$$
M_{\pi}=\sum_{i=1}^{N}(\bar{h}(i)-\underline{h}(i))=\sum_{i=1}^{N} \Delta h(i) .
$$

It is clear that $M_{\pi}$ is an upper bound on $M$ for all plants in the family $\pi$ :

$$
M_{\pi} \geq M, \forall h \in \pi
$$

In the rest of this paper, the nominal model $\hat{h}$ is assumed to be in the plants family $\pi$.

\subsection{SISO DMC algorithm based on FIR model}

In general, the DMC algorithm is based on a step response model. To apply the techniques in [14] to solve the robust stability problem of the DMC controller, Dai reconstructed the DMC algorithm based on an 
FIR model in [15].

The aim of DMC algorithms is to find a control series $\{\Delta u(t), \Delta u(t+1), \cdots, \Delta u(t+m-1)\}$ that minimizes the objective function

$$
\Phi(t)=\sum_{j=1}^{p} q_{j} \cdot(r(t)-\hat{y}(t+j))^{2}+\sum_{i=1}^{m} r_{i} \cdot \Delta u^{2}(t+i-1)
$$

In this representation, where $r(t)$ is the reference input, $q_{j}$ and $r_{i}$ are the output error weights and input suppression weights respectively, and constants $p$ and $m$ denote the prediction horizon and the control horizon, normally we have $N \geq p \geq m$.

The output error series is defined as

$$
e(t+j)=r(t)-\hat{y}(t+j)=\hat{e}(t+j)-\hat{y}_{f}(t+j)
$$

where $\hat{e}(t+j)$ satisfies

$$
\hat{e}(t+j)=r(t)-\hat{y}_{p}(t+j)-\hat{d}(t)
$$

From (12) and (13) vector equations can be obtained as follows:

$$
\begin{aligned}
& \hat{\mathbf{y}}_{\mathbf{f}}=\mathbf{H} \cdot \mathbf{u}, \mathbf{e}=\hat{\mathbf{e}}-\mathbf{H} \cdot \mathbf{u} \\
& \Delta \mathbf{u}=\mathbf{G} \cdot \mathbf{u}-\mathbf{b} \cdot u(t-1)
\end{aligned}
$$

where

$$
\hat{\mathbf{y}}_{\mathbf{f}}=\left[\begin{array}{c}
\hat{y}_{f}(t+1) \\
\vdots \\
\hat{y}_{f}(t+p)
\end{array}\right], \mathbf{u}=\left[\begin{array}{c}
u(t) \\
\vdots \\
u(t+m-1)
\end{array}\right], \mathbf{H}=\left[\begin{array}{cccc}
\hat{h}(1) & 0 & \cdots & 0 \\
\hat{h}(2) & \hat{h}(1) & \ddots & \vdots \\
\vdots & \vdots & \ddots & 0 \\
\hat{h}(m) & \hat{h}(m-1) & \cdots & \hat{h}(1) \\
\vdots & \vdots & & \vdots \\
\hat{h}(p) & \hat{h}(p-1) & \ldots & \sum_{i=1}^{p-m+1} \hat{h}(i)
\end{array}\right], \quad \mathbf{e}=\left[\begin{array}{c}
e(t+1) \\
\vdots \\
e(t+p)
\end{array}\right],
$$


$\hat{\mathbf{e}}=\left[\begin{array}{c}\hat{e}(t+1) \\ \vdots \\ \hat{e}(t+p)\end{array}\right], \Delta \mathbf{u}=\left[\begin{array}{c}\Delta u(t) \\ \vdots \\ \Delta u(t+m-1)\end{array}\right], \mathbf{G}=\left[\begin{array}{cccc}1 & 0 & \cdots & 0 \\ -1 & 1 & \ddots & \vdots \\ \vdots & \ddots & \ddots & 0 \\ 0 & \cdots & -1 & 1\end{array}\right], \mathbf{b}=\left[\begin{array}{llll}1 & 0 & \cdots & 0\end{array}\right]^{\mathrm{T}}$

Let $\mathbf{Q}=\operatorname{diag}\left(q_{1}, \cdots, q_{p}\right), \mathbf{R}=\operatorname{diag}\left(r_{1}, \cdots, r_{m}\right)$, then the objective function (11) can be rewritten as

$$
\Phi(t)=(\hat{\mathbf{e}}-\mathbf{H} \cdot \mathbf{u})^{\mathrm{T}} \mathbf{Q}(\hat{\mathbf{e}}-\mathbf{H} \cdot \mathbf{u})+(\mathbf{G} \cdot \mathbf{u}-\mathbf{b} \cdot u(t-1))^{\mathrm{T}} \mathbf{R}(\mathbf{G} \cdot \mathbf{u}-\mathbf{b} \cdot u(t-1)) .
$$

The corresponding optimal solution is $\mathbf{u}=\left(\mathbf{H}^{\mathrm{T}} \mathbf{Q} \mathbf{H}+\mathbf{G}^{\mathrm{T}} \mathbf{R} \mathbf{G}\right)^{-1}\left(\mathbf{H}^{\mathrm{T}} \mathbf{Q} \hat{\mathbf{e}}+\mathbf{G}^{\mathrm{T}} \mathbf{R} \mathbf{b} \cdot u(t-1)\right)$. Note that only the first term $u(t)$ of $\mathbf{u}$ is employed in the DMC algorithm, thus we have

$$
u(t)=\mathbf{k}_{\mathbf{e}} \cdot \hat{\mathbf{e}}+k_{u} \cdot u(t-1)
$$

where

$$
\begin{aligned}
& \mathbf{k}_{\mathbf{e}}=\mathbf{b}^{\mathrm{T}}\left(\mathbf{H}^{\mathrm{T}} \mathbf{Q H}+\mathbf{G}^{\mathrm{T}} \mathbf{R} \mathbf{G}\right)^{-1} \mathbf{H}^{\mathrm{T}} \mathbf{Q}=\left[k_{e 1} \cdots k_{e p}\right] \\
& k_{u}=\mathbf{b}^{\mathrm{T}}\left(\mathbf{H}^{\mathrm{T}} \mathbf{Q H}+\mathbf{G}^{\mathrm{T}} \mathbf{R} \mathbf{G}\right)^{-1} \mathbf{G}^{\mathrm{T}} \mathbf{R} \mathbf{b} .
\end{aligned}
$$

Substituting (1) - (3) and (13) into (16), a difference equation describing the DMC controller can be given by

$$
u(t)=k_{u} \cdot u(t-1)+\sum_{i=1}^{N} k_{i} \cdot u(t-i)+k_{r} \cdot r(t)
$$

where

$$
k_{r}=\sum_{j=1}^{p} k_{e j}, k_{i}=\sum_{j=1}^{p} k_{e j}[\hat{h}(i)-h(i)-\hat{h}(i+j)], i=1,2, \cdots, N
$$

Therefore, the stability of the DMC algorithm can be analyzed from (17) directly.

\section{Main Results}

In this section, a similar procedure is used to analyze the stability of a remote SISO DMC algorithm in NCSs based on an FIR model.

\subsection{Control system structure}

Any plantwide process control system involves four levels of control. From the bottom up the four levels 
are: the process protection level, the basic regulatory control level, the advanced control level, and the overall plant optimization level. If the network is connected with the regulatory control level and the advanced control level is located at the remote side to cooperate with other plants, the control system will have a two level hierarchy. This two level control structure was widely used in industrial applications. The random Internet transmission delay exists at both the feed-forward and feedback channels for symmetric communication. There are some assumptions we need to make before we study the NCSs in the discrete time domain. In order to have appropriate updates from the sensor side to the controller side we must ensure that both are synchronized, in the sense that both will carry out their respective tasks at the same time. Moreover, updates will be carried out at some of those time instants. Maintaining global clock synchronization in distributed systems is a challenging task, but recent work has shown remarkably small synchronization errors even in large sensor networks [35]. Fig. 2 illustrates the control structure of this scenario. We use $T_{\text {local }}$ and $T_{\text {remote }}$ to denote the sampling intervals of the local controller and the remote controller respectively. To meet the requirement of synchronization, $T_{\text {remote }}$ is chosen as a multiple of $T_{\text {local }}$.

Without loss of generality, we assume that the local sampling interval satisfies $T_{\text {local }}=1$. The networked-induced delays in the feedback and feed-forward channels are denoted by $\tau_{s c}$ and $\tau_{c a}$. Although these delays are unknown, a common assumption which is often made is that these delays have upper bounds, which are denoted by an positive integer $\bar{\tau}_{s c}$ and $\bar{\tau}_{c a}$. Note that the two integers are the numbers of sampling intervals of the maximum delays. We assume that these two integers satisfy $\bar{\tau}_{s c}, \bar{\tau}_{c a}<p$.

To simplify the analysis, we assume that the time-varying controller-actuator delay is converted to the constant maximum delay $\bar{\tau}_{c a}$ by using a receiving buffer, i.e. control signal $u^{*}(t)$ satisfies 
$u^{*}(t)=u\left(t-\bar{\tau}_{c a}\right)$. Therefore, the inner control loop forms a new stable "local plant" including the constant delay component and the locally stabilized plant. The remote controller is designed as a DMC controller to perform an advanced control scheme. It should be pointed out that in this DMC algorithm the prediction model is different from Eq. (2), since the plant output feedback $y(t)$ cannot arrive at the remote controller in time. A clear picture of these assumptions is depicted as Fig. 2.

In Fig. 2, $y\left(t^{*}\right)$ denotes the measured feedback via network at time $t$, where $t^{*}$ is mostly an integer that can be regarded as a function of $t$. It is apparent that $t^{*}$ satisfies $t^{*}(t)<t$. It should be pointed out that here $t^{*}$ is not necessarily the timestamp of the latest received data. Note that using the latest received data in the controller leads to a time-varying delay problem, which might complicate the stability analysis.

To apply the DMC algorithm based on the FIR model in this system structure, a general procedure similar to that described in section 2 is presented as follows. Note that some changes should be made in the original procedure due to the networked-induced delay and the choice of the sampling interval $T_{\text {remote }}$.

1) The stable linear delay local plant in Fig. 2 is described by the following FIR model:

$$
y(t)=\sum_{i=1}^{N} h(i) u^{*}(t-i)=\sum_{i=1}^{N} h(i) u\left(t-i-\bar{\tau}_{c a}\right), t \in \mathbf{Z} .
$$

Note that the sampling interval is $T_{\text {local }}$ in this model.

2) The predicted future values of the plant output are given by

$$
\begin{aligned}
\hat{y}(t+j) & =\sum_{i=1}^{N} \hat{h}(i) u^{*}(t+j-i)+\hat{d}\left(t^{*}\right) \\
& =\sum_{i=1}^{N} \hat{h}(i) u\left(t+j-i-\bar{\tau}_{c a}\right)+\hat{d}\left(t^{*}\right) \\
& =\hat{y}_{f}^{*}(t+j)+\hat{y}_{p}^{*}(t+j)+\hat{d}\left(t^{*}\right)
\end{aligned}
$$

where 


$$
\begin{gathered}
\hat{y}_{f}^{*}(t+j)=\sum_{i=1}^{j-\bar{\tau}_{c a}} \hat{h}(i) u\left(t+j-i-\bar{\tau}_{c a}\right), \\
\hat{y}_{p}^{*}(t+j)=\sum_{i=j-\bar{\tau}_{c a}+1}^{N} \hat{h}(i) u\left(t+j-i-\bar{\tau}_{c a}\right), \\
\hat{d}\left(t^{*}\right)=y\left(t^{*}\right)-\hat{y}\left(t^{*}\right) .
\end{gathered}
$$

3) Introduce a new output error series

$$
e(t+j)=r(t)-\hat{y}(t+j)=\hat{e}^{*}(t+j)-\hat{y}_{f}^{*}(t+j)
$$

which is different from (12) with

$$
\hat{e}^{*}(t+j)=r(t)-\hat{y}_{p}^{*}(t+j)-\hat{d}\left(t^{*}\right)
$$

From (23) and (24) the new vector equations can be obtained as follows:

$$
\hat{\mathbf{y}}_{\mathbf{f}}^{*}=\mathbf{H}^{*} \cdot \mathbf{u}, \mathbf{e}^{*}=\hat{\mathbf{e}}^{*}-\mathbf{H}^{*} \cdot \mathbf{u}
$$

where

$$
\begin{aligned}
\hat{\mathbf{y}}_{\mathbf{f}}^{*}=\left[\begin{array}{c}
\hat{y}_{f}^{*}(t+1) \\
\vdots \\
\hat{y}_{f}^{*}(t+p)
\end{array}\right], \mathbf{H}^{*}=\left[\begin{array}{cccc}
0 & 0 & \cdots & 0 \\
\vdots & & & \vdots \\
0 & 0 & \cdots & 0 \\
\hat{h}(1) & 0 & \cdots & 0 \\
\hat{h}(2) & \hat{h}(1) & \ddots & \vdots \\
\vdots & \vdots & \ddots & 0 \\
\hat{h}(m) & \hat{h}(m-1) & \cdots & \hat{h}(1) \\
\vdots & \vdots & & \vdots \\
\hat{h}\left(p-\bar{\tau}_{c a}\right) & \hat{h}\left(p-\bar{\tau}_{c a}-1\right) & \cdots & \sum_{i=1}^{p-m+1-\bar{\tau}_{c a}} \\
& &
\end{array} \hat{h}^{\prime}(i)\right. \\
\hat{\mathbf{e}}^{*}=\left[\begin{array}{c}
\hat{e}^{*}(t+1) \\
\vdots \\
\hat{e}^{*}(t+p)
\end{array}\right] .
\end{aligned}
$$

4) The corresponding objective function of DMC and its optimal solution are

$$
\Phi(t)=\left(\hat{\mathbf{e}}^{*}-\mathbf{H}^{*} \cdot \mathbf{u}\right)^{\mathrm{T}} \mathbf{Q}\left(\hat{\mathbf{e}}^{*}-\mathbf{H}^{*} \cdot \mathbf{u}\right)+(\mathbf{G} \cdot \mathbf{u}-\mathbf{b} \cdot u(t-1))^{\mathrm{T}} \mathbf{R}(\mathbf{G} \cdot \mathbf{u}-\mathbf{b} \cdot u(t-1))
$$

and 


$$
\mathbf{u}=\left(\mathbf{H}^{* \mathrm{~T}} \mathbf{Q} \mathbf{H}^{*}+\mathbf{G}^{\mathrm{T}} \mathbf{R} \mathbf{G}\right)^{-1}\left(\mathbf{H}^{* \mathrm{~T}} \mathbf{Q} \hat{\mathbf{e}}^{*}+\mathbf{G}^{\mathrm{T}} \mathbf{R} \mathbf{b} \cdot u(t-1)\right)
$$

Therefore, the first item of $\mathbf{u}$ is given by

$$
u(t)=\mathbf{k}_{\mathbf{e}}^{*} \cdot \hat{\mathbf{e}}^{*}+k_{u}^{*} \cdot u(t-1)=\sum_{j=1}^{p}{k_{e j}}^{*} \hat{e}^{*}(t+j)+k_{u}^{*} \cdot u(t-1)
$$

where

$$
\begin{aligned}
\mathbf{k}_{\mathbf{e}}{ }^{*} & =\mathbf{b}^{\mathrm{T}}\left(\mathbf{H}^{* \mathrm{~T}} \mathbf{Q} \mathbf{H}^{*}+\mathbf{G}^{\mathrm{T}} \mathbf{R} \mathbf{G}\right)^{-1} \mathbf{H}^{* \mathrm{~T}} \mathbf{Q}=\left[k_{e 1}{ }^{*} \cdots k_{e p}{ }^{*}\right] \\
k_{u}{ }^{*} & =\mathbf{b}^{\mathrm{T}}\left(\mathbf{H}^{* \mathrm{~T}} \mathbf{Q} \mathbf{H}^{*}+\mathbf{G}^{\mathrm{T}} \mathbf{R} \mathbf{G}\right)^{-1} \mathbf{G}^{\mathrm{T}} \mathbf{R} \mathbf{b} .
\end{aligned}
$$

5) Substituting (18), (19), (21), (22) and (24) into (26) leads to

$$
\begin{aligned}
u(t)= & k_{r}^{*} \cdot r(t)+k_{u}^{*} \cdot u(t-1)-\sum_{j=1}^{p}{k_{e j}}^{*} \cdot\left[\sum_{i=j-\bar{\tau}_{c a}+1}^{N} \hat{h}(i) u\left(t+j-i-\bar{\tau}_{c a}\right)\right] \\
& +k_{r}{ }^{*} \cdot\left[\sum_{i=1}^{N}[\hat{h}(i)-h(i)] u\left(t^{*}-i-\bar{\tau}_{c a}\right)\right]
\end{aligned}
$$

The coefficient $k_{r}{ }^{*}$ satisfies $k_{r}{ }^{*}=\sum_{j=1}^{p} k_{e j}{ }^{*}$. From now on, the stability problem of this DMC algorithm is reduced to the stability problem of a difference Eq. (27).We call the algorithm derived from (18) to (27) the remote DMC controller in our proposed NCS structure.

In solving the concomitant issues posed by the network, there are several methods to determine the actual time $t^{*}$ of measured feedback $y$. In the following sections, we discuss two of them: the maximum delayed output feedback method and the multi-rate sampling method.

\subsection{Maximum delayed output feedback method}

In this case, we assume a single-rate control scheme in which the remote controller's sampling interval $T_{\text {remote }}$ is equal to the local one $T_{\text {local }}$. As mentioned above, the networked-induced feedback delay is unknown but has an upper bound, which is denoted by an integer $\bar{\tau}_{s c}$. Note that $\bar{\tau}_{s c}$ is the number of sampling intervals of the maximum delay. Therefore the remote DMC controller must have received the feedback data 
$y\left(t-\bar{\tau}_{s c}\right)$ at time $t$. Furthermore, $y\left(t-\bar{\tau}_{s c}\right)$ is used to form the additional term $\hat{d}\left(t^{*}\right)$ by selecting $t^{*}=t-\bar{\tau}_{s c}$ in (22). This can be easily implemented by designing a $\bar{\tau}_{s c}$-unit data buffer to hold arrived feedback data at the remote DMC controller.

It follows that Eq. (27) can be written as

$$
\begin{aligned}
u(t)= & k_{r}^{*} \cdot r(t)+k_{u}^{*} \cdot u(t-1)-\sum_{j=1}^{p} k_{e j}^{*} \cdot\left[\sum_{i=j-\bar{\tau}_{c a}+1}^{N} \hat{h}(i) u\left(t+j-i-\bar{\tau}_{c a}\right)\right] \\
& +k_{r}^{*} \cdot\left[\sum_{i=1}^{N}[\hat{h}(i)-h(i)] u\left(t-\bar{\tau}_{s c}-i-\bar{\tau}_{c a}\right)\right]
\end{aligned}
$$

A simple criterion to investigate the stability of the remote DMC controller is obtained as follows.

Lemma 1. [28] (Jury's dominant coefficient lemma) Consider a characteristic equation $A\left(z^{-1}\right)=\sum_{i=0}^{\infty} a_{i} z^{-i}$. If condition $\sum_{i=0}^{\infty}\left|a_{i}\right|<1$ is satisfied, then the discrete system described by the characteristic equation is stable.

Theorem 1. When using the maximum delayed output feedback method, the remote DMC controller (28) is stable for all stable plants in the family $\pi$ if

$$
\left|k_{u}^{*}\right|+\sum_{i=1}^{N+\bar{\tau}_{c a}}\left|\sum_{j=1}^{p} k_{e j}^{*} \cdot \hat{h}\left(i+j-\bar{\tau}_{c a}\right)\right|+\left|k_{r}^{*}\right| \cdot M_{\pi}<1
$$

Proof. When $\bar{\tau}_{s c}<N$, to discuss the stability of difference Eq. (28) we should investigate

$$
\begin{aligned}
& u(t)=k_{u}^{*} \cdot u(t-1)-\sum_{j=1}^{p} k_{e j}^{*} \cdot\left[\sum_{i=j-\bar{\tau}_{c a}+1}^{N} \hat{h}(i) u\left(t+j-i-\bar{\tau}_{c a}\right)\right]+ \\
& k_{r}^{*} \cdot\left[\sum_{i=1}^{N}[\hat{h}(i)-h(i)] u\left(t-\bar{\tau}_{s c}-i-\bar{\tau}_{c a}\right)\right] .
\end{aligned}
$$

Because $\hat{h}(i)=h(i)=0$ for any $i>N$ or $i<1$, the above equation can be rewritten as 


$$
\begin{aligned}
& u(t)=k_{u}^{*} \cdot u(t-1)-\sum_{j=1}^{p} k_{e j}^{*} \cdot\left[\sum_{i=1}^{N+\bar{\tau}_{c a}} \hat{h}\left(i+j-\bar{\tau}_{c a}\right) u(t-i)\right]+ \\
& k_{r}^{*} \cdot\left\{\sum_{i=1}^{N}[\hat{h}(i)-h(i)] u\left(t-\bar{\tau}_{s c}-i-\bar{\tau}_{c a}\right)\right\} \\
& =k_{u}^{*} \cdot u(t-1)-\sum_{i=1}^{N+\bar{\tau}_{c a}}\left[\sum_{j=1}^{p} k_{e j}^{*} \cdot \hat{h}\left(i+j-\bar{\tau}_{c a}\right)\right] u(t-i)+k_{r}^{*} \cdot\left\{\sum_{i=1}^{N}[\hat{h}(i)-h(i)] \mu\left(t-\bar{\tau}_{s c}-i-\bar{\tau}_{c a}\right)\right\} \\
& =k_{u}^{*} \cdot u(t-1)-\sum_{i=1}^{\bar{\tau}_{c a}+\bar{\tau}_{s c}}\left[\sum_{j=1}^{p} k_{e j}^{*} \cdot \hat{h}\left(i+j-\bar{\tau}_{c a}\right)\right] u(t-i)+ \\
& k_{r}^{*} \cdot\left\{\sum_{i=\bar{\tau}_{c a}+\bar{\tau}_{s c}+1}^{N+\bar{\tau}_{c a}}\left[\hat{h}\left(i-\bar{\tau}_{s c}-\bar{\tau}_{c a}\right)-h\left(i-\bar{\tau}_{s c}-\bar{\tau}_{c a}\right)-\frac{1}{k_{r}^{*}} \sum_{j=1}^{p} k_{e j}^{*} \cdot \hat{h}\left(i+j-\bar{\tau}_{c a}\right)\right] u(t-i)\right\}+ \\
& k_{r}^{*} \cdot\left\{\sum_{i=N+\bar{\tau}_{c a}+1}^{N+\bar{\tau}_{c a}+\bar{\tau}_{s c}}\left[\hat{h}\left(i-\bar{\tau}_{s c}-\bar{\tau}_{c a}\right)-h\left(i-\bar{\tau}_{s c}-\bar{\tau}_{c a}\right)\right] u(t-i)\right\}
\end{aligned}
$$

It follows from (29) and the definition of $M_{\pi}$ that

$$
\begin{aligned}
& \left|k_{u}^{*}\right|+\sum_{i=1}^{\bar{\tau}_{s c}+\bar{\tau}_{c a}}\left|\sum_{j=1}^{p} k_{e j}{ }^{*} \cdot \hat{h}\left(i+j-\bar{\tau}_{c a}\right)\right|+\left|k_{r}{ }^{*}\right| \cdot \sum_{i=N+\bar{\tau}_{c a}+1}^{N+\bar{c}_{c a}+\bar{\tau}_{s c}}\left|\hat{h}\left(i-\bar{\tau}_{s c}-\bar{\tau}_{c a}\right)-h\left(i-\bar{\tau}_{s c}-\bar{\tau}_{c a}\right)\right|+ \\
& \left|k_{r}^{*}\right| \cdot \sum_{i=\bar{\tau}_{c a}+\bar{\tau}_{s c}+1}^{N+\bar{\tau}_{c a}}\left|\hat{h}\left(i-\bar{\tau}_{s c}-\bar{\tau}_{c a}\right)-h\left(i-\bar{\tau}_{s c}-\bar{\tau}_{c a}\right)-\frac{1}{k_{r}{ }^{*}} \sum_{j=1}^{p} k_{e j}{ }^{*} \cdot \hat{h}\left(i+j-\bar{\tau}_{c a}\right)\right| \leq \\
& \left|k_{u}{ }^{*}\right|+\sum_{i=1}^{\bar{\tau}_{s c}+\bar{\tau}_{c a}}\left|\sum_{j=1}^{p} k_{e j}{ }^{*} \cdot \hat{h}\left(i+j-\bar{\tau}_{c a}\right)\right|+\sum_{i=\bar{\tau}_{c a}+\bar{\tau}_{s c}+1}^{N+\bar{\tau}_{c a}}\left|\sum_{j=1}^{p} k_{e j}{ }^{*} \cdot \hat{h}\left(i+j-\bar{\tau}_{c a}\right)\right|+\left|k_{r}{ }^{*}\right| \cdot \sum_{i=1}^{N}|\hat{h}(i)-h(i)| \leq \\
& \left|k_{u}^{*}\right|+\sum_{i=1}^{N+\bar{\tau}_{c a}}\left|\sum_{j=1}^{p} k_{e j}^{*} \cdot \hat{h}\left(i+j-\bar{\tau}_{c a}\right)\right|+\left|k_{r}{ }^{*}\right| \cdot M_{\pi}<1 .
\end{aligned}
$$

When $\bar{\tau}_{\text {sc }} \geq N$, we can obtain the same condition in a similar way.

In terms of Jury’s dominant coefficient lemma, the remote DMC controller is therefore stable.

This theorem leads to an interesting conclusion that the feedback delay has no effect on the stability of the remote DMC controller. However, a long delay may deteriorate other performances in the closed-loop system.

\subsection{Multi-rate sampling method}


The study of multi-rate sampled-data systems has been a great success in the past several years; see Izadi et al. [29], Hu et al. [30], Wang et al. [11] and Izadi et al. [31]. Using the multi-rate method, Lin et al. [32] considered stability and disturbance attenuation issues for a class of NCSs in the framework of switched systems when random delay is less than one sample period. Georgiev et al. [33] used multipoint packets to reduce network traffic and computation time. Yang et al. [7] investigated the potential of using the multi-rate control scheme and the time-delay compensation to overcome Internet transmission delay.

In this section we discuss a multi-rate sampling method in which the local and remote controllers are running at different frequencies. The local controller at the lower level in the hierarchy is running at a higher frequency to stabilize the plant, the remote controller at the higher level at a lower frequency to reduce the communication load and increase the possibility of receiving data on time. We call the local controller the fast controller and the remote controller the slow controller.

The two sampling intervals for the fast and slow controllers are chosen as:

$$
T_{\text {remote }}=T \cdot T_{\text {local }}, T \in\{2,3,4, \cdots\}
$$

Without loss of generality, we let $T_{\text {local }}=1$ so that the remote DMC controller receives new data only at instants $\{T, 2 T, 3 T, \cdots\}$. Considering the networked-induced delay, the actual timestamp of the feedback data at time $t$ can be given by

$$
t^{*}(t)=l T-\bar{\tau}_{s c}, l=0,1,2, \cdots, t \in\{l T, I T+1, \cdots l T+T-1\}
$$

where the output feedback delay $\bar{\tau}_{s c}$ is assumed to satisfy $\bar{\tau}_{s c}<T$.

Substituting (31) into (27), it follows that 


$$
\begin{aligned}
& u(t)=k_{r}^{*} \cdot r(t)+k_{u}^{*} \cdot u(t-1)-\sum_{j=1}^{p} k_{e j}^{*} \cdot\left[\sum_{i=j-\tau_{c a}+1}^{N} \hat{h}(i) u\left(t+j-i-\bar{\tau}_{c a}\right)\right]+ \\
& k_{r}^{*} \cdot\left[\sum_{i=1}^{N}[\hat{h}(i)-h(i)] u\left(l T-\bar{\tau}_{s c}-i-\bar{\tau}_{c a}\right)\right], \quad t \in\{l T, I T+1, \cdots l T+T-1\} .
\end{aligned}
$$

To discuss the stability of the remote DMC controller (32), the following linear periodic difference system should be investigated:

$$
\begin{aligned}
& u(t)=k_{u}^{*} \cdot u(t-1)-\sum_{j=1}^{p} k_{e j}^{*} \cdot\left[\sum_{i=j-\bar{\tau}_{c a}+1}^{N} \hat{h}(i) u\left(t+j-i-\bar{\tau}_{c a}\right)\right]+ \\
& k_{r}^{*} \cdot\left[\sum_{i=1}^{N}[\hat{h}(i)-h(i)] u\left(t-m-\bar{\tau}_{s c}-i-\bar{\tau}_{c a}\right)\right], t=l T+m, m \in\{0,1, \cdots, T-1\} .
\end{aligned}
$$

We start by studying the stability of the switching difference system given by

$$
\begin{aligned}
& u(t)=a_{\sigma(t), 1} u(t-1)+a_{\sigma(t), 2} u(t-2)+\cdots+a_{\sigma(t), M} u(t-M), \\
& \sigma(t) \in\{0,1, \cdots, T-1\}
\end{aligned}
$$

where $\sigma(t)$ denotes the sequence of switching modes. The $i$-th coefficient $a_{\sigma(t), i}$ is time-varying and should be chosen from $T$ possible values. The following lemma gives a simple stability criterion to test the stability of system (34).

Lemma 2. Switching difference system (34) is uniformly stable if inequality

$$
\left|a_{\sigma(t), 1}\right|+\left|a_{\sigma(t), 2}\right|+\cdots+\left|a_{\sigma(t), M}\right|<1
$$

is satisfied for all $\sigma(t)$.

Proof. This lemma can be easily proved using a similar method as presented in Theorem 5.4.1 of [34].

The following theorem provides simple stability criterion for the remote DMC controller (32).

Theorem 2. When using the multi-rate sampling method, the remote DMC controller (33) is uniformly stable for all stable plants in the family $\pi$ if inequality (29) is satisfied.

Proof. System (33) can be rewritten as a switching difference system given by 


$$
\begin{aligned}
& u(t)=a_{\sigma(t), 1} \cdot u(t-1)+a_{\sigma(t), 2} \cdot u(t-2)+\cdots+a_{\sigma(t), T-1+\bar{\tau}_{s c}+\bar{\tau}_{c a}+N} \cdot u\left(t-T+1-\bar{\tau}_{s c}-\bar{\tau}_{c a}-N\right), \\
& \sigma(t) \in\{0,1, \cdots, T-1\}
\end{aligned}
$$

where $a_{\sigma(t), 1}=k_{u}^{*}$ and other coefficients are periodically time-varying.

It follows from (29) that for all possible $\sigma(t)$

$$
\begin{aligned}
& \left|a_{\sigma(t), 1}\right|+\left|a_{\sigma(t), 2}\right|+\cdots+\left|a_{\sigma(t), T-1+\bar{\tau}_{s c}+\bar{\tau}_{c a}+N}\right|< \\
& \left|k_{u}{ }^{*}\right|+\sum_{i=1}^{N+\bar{\tau}_{c a}}\left|\sum_{j=1}^{p}{k_{e j}}^{*} \cdot \hat{h}\left(i+j-\bar{\tau}_{c a}\right)\right|+\left|k_{r}{ }^{*}\right| \cdot \sum_{i=1}^{N}|\hat{h}(i)-h(i)| \leq \\
& \left|k_{u}{ }^{*}\right|+\sum_{i=1}^{N+\bar{\tau}_{c a}}\left|\sum_{j=1}^{p}{k_{e j}}^{*} \cdot \hat{h}\left(i+j-\bar{\tau}_{c a}\right)\right|+\left|k_{r}{ }^{*}\right| \cdot M_{\pi}<1 .
\end{aligned}
$$

According to Lemma 2, the remote DMC controller (32) is then uniformly stable.

The condition of this theorem is also the same as theorem 1, which indicates that multiple sampling rate has no effect on the stability.

Corollary 1. If maximum delayed feedback or multi-rate sampling method is used to design a remote DMC controller (28), the maximum allowable uncertainty of plant family $\pi$ is

$$
M_{\max }=\left(1-\left|k_{u}{ }^{*}\right|-\sum_{i=1}^{N+\bar{\tau}_{c a}}\left|\sum_{j=1}^{p} k_{e j}{ }^{*} \cdot \hat{h}\left(i+j-\bar{\tau}_{c a}\right)\right|\right) /\left|k_{r}{ }^{*}\right| \cdot
$$

Proof. According to theorem 1 and 2, the conclusion is apparent.

\section{Simulation Study}

A family of plants of both gain and dead time uncertainties were investigated to demonstrate our proposed theory. Consider the plant

$$
G(s)=\frac{Y(s)}{U(s)}=\frac{K_{1}}{s^{2}+2 s+1} e^{-\theta s}
$$

with a zero-order hold and where $T$ is 0.2 sec. The gain $K_{1}$ of the model is unknown but lies within the bound $[0.9,1.1]$. The dead time $\theta$ is also unknown but lies within the bound $[0,0.3]$. 
All the simulation programs were implemented in the MATLAB 6.5 environment. The results of pulse response for the plant family (37) are shown by Fig. 3. Choosing $N=40$, the maximum total mismatch defined by (9) is 2.4596, which can be easily computed using the two bounds shown in Fig. 3.

In the simulation study we use a nominal model (37) with $K_{1}=1, \theta=0$ and an actual model with $K_{1}=1, \theta=0.2$. Weight matrices are chosen as $Q=2 \times I_{p}, R=0.2$. It turns out that the allowable uncertainty $M_{\max }$ depends significantly on parameter $p$. Normally a larger $p$ results in a larger allowable uncertainty. To implement the remote DMC controller given by (28), we chose $p=30$ and $m=1$. Hence, the coefficients of controller and the maximum allowable uncertainty are obtained as follows:

$$
k_{r}^{*}=0.2607, k_{u}^{*}=0.00034, M_{\max }=2.6985 .
$$

Because the maximum allowable uncertainty is bigger than the maximum total mismatch of plant family (36), the obtained remote DMC controller can then robustly stabilize any plant in this family. In the case of using the maximum delayed feedback method, two maximum delays were investigated in the simulation. Assuming the reference input $r(t)$ is a step function and the maximum feed-forward delay $\bar{\tau}_{c a}=1 \mathrm{~s}$, responses are depicted in Fig. 4 for closed-loop systems with maximum feedback delay $\bar{\tau}_{s c}=1 \mathrm{~s}, \bar{\tau}_{s c}=2 \mathrm{~s}$ and $\bar{\tau}_{s c}=4 \mathrm{~s}$ respectively. Note that although the output of the remote DMC controller is retarded by the transmission delay significantly, the closed-loop system is still asymptotically stable. On the other hand, large transmission delays lead to a long settling time of plant response.

In the case of using the multi-rate sampling method, two sampling rates, $T_{\text {remote }}=10$ and $T_{\text {remote }}=25$, were investigated in the simulation. Assuming the reference input $r(t)$ is a step function and the 
maximum delays $\bar{\tau}_{c a}=1 \mathrm{~s}, \bar{\tau}_{s c}=2 \mathrm{~s}$, responses are depicted in Fig. 5. A nominal case of $T_{\text {remote }}=1$ is also illustrated in the figure. As we can see, in all the situations the closed-loop system is asymptotic stable, which confirms our proposed theoretical results.

\section{Conclusions}

Introducing networks into control systems brings many new issues. One of the major problems is the transmission delay caused by the data exchange through communication channels. Design methods and theoretical analysis in a normal feedback control system are very different from those in a NCS.

The main contribution of this study is to obtain some simple stability criteria for a remote DMC controller to control networked processes, which are stabilized by a local controller. In our two-level hierarchy of NCS, delayed feedback sensory data is used to design a novel remote DMC controller. We choose two methods to cope with the delayed data. A simple inequality is obtained to judge the robust stability of the proposed controller. It turns out that the robust stability of the new DMC controller has nothing to do with the feedback delay in both cases. However, long transmission delay may deteriorate other performances in the closed-loop system, such as settling time and overshoot in the plant responses.

Our future work might be the extension of the proposed theory in the Multiple-In-Multiple-Out (MIMO) cases of networked control systems. Furthermore, the networked MPC synthesis problem for nonlinear uncertain processes needs to be further investigated.

\section{Acknowledgement}

This work was partly supported by NSF China under Grant No. 69974017, 60274020, 60603006, 
60674105, 60340420431.

\section{References}

[1] J. Nilsson, Real-time control systems with delays, Ph.D. dissertation,Dept. Automatic Control, Lund Institute of Technology, Lund, Sweden, (1998)

[2] A. Hassibi, S.P. Boyd and J.P. How, Control of asynchronous dynamical systems with rate constraints on events, in: Proceedings of IEEE Conf. Decision and Control, Phoenix, AZ, 1999, pp. 1345-1351

[3] W. Zhang, M.S. Branicky and S.M. Phillips, Stability of networked control systems, IEEE Contr. Syst. Mag., 19 (2001) 84-99

[4] P.V. Zhivoglyadov, R.H. Middleton, Networked control design for linear systems, Automatica, (2003) $743-750$

[5] L.A. Montestruque, P.J. Antsaklis, On the model-based control of networked systems, Automatica, 39 (2003) $1837-1843$

[6] L.Q. Zhang, T.W. Chen, and B. Huang, A new method for stabilization of network control systems with random delays, IEEE Transactions on Automatic Control, 50(8) (2005) 1177-1181

[7] L. Yang, and S.H. Yang, Multirate control in Internet-based control systems, IEEE Transactions on Systems, Man, and Cybernetics, Part C: Applications and Reviews, 37(2) (2007) 185-192

[8] S.H. Yang, X. Chen, and J.L. Alty, Design issues and implementation of Internet-based process control systems, Control Eng. Practice, 11, (2003) 709-720

[9] S.H. Yang, X. Zuo, and L. Yang, Control system for Internet-enabled arm robots, Lecture Notes in AI, 3029, (2004) 663-672 
[10] S. S. Hu and Q. X. Zhu, Stochastic optimal control and analysis of stability of networked control systems with long delay. Automatica, 39 (2003) 1877-1884

[11] J. Wang, T. Chen and B. Huang. Multirate sampled-data systems: computing fast-rate models. Journal of process control, 14 (2004) 79-88

[12]J. Huang, Z.H. Guan and Z.D. Wang, Stability of networked control systems based on model of discrete-time interval system with uncertain delay, Dynamics of Continuous, Discrete and Impulsive Systems Series B: Applications \& Algorithms, 11a, (2004) 35-44

[13]C.R. Cutler, B.L. Ramaker, Dynamic matrix control—a computer control algorithm, In: Proceedings. Joint American Control Conf., San Francisco, (1980)

[14] T.A. Badgwell, Robust stability conditions for SISO model predictive control algorithms, Automatica, 33, (1997) 1357-1361

[15] L.K. Dai, Z.Q. Cheng, Robust stability conditions for SISO dynamic matrix control, Developments in Chemical Engineering and Mineral Processing, 9(1-2), 49-56, (2001)

[16] J.R. Webber and Y.P. Gupta, Robust and nominal stability conditions for a simplified model predictive controller, ISA Transactions, 45(1) (2006) 77-86

[17] A.Y. Torgashov, Robust-stabilizing controller horizons on the basis of predictive model with truncated pulse transient function, Automation and Remote Control, 68 (7) (2007) 1201-1212

[18] K.R. Muske, T.A. Badgwell, Disturbance modeling for offset-free linear model predictive control, Journal of Process Control, 12 (2002) 617-632.

[19] A. Zheng, Robust stability analysis of constrained model predictive control, Journal of Process Control, 9 (1999) 271-278. 
[20] L.O. Santos, L.T. Biegler, A tool to analyze robust stability for model predictive controllers, Journal of Process Control, 9 (1999) 233-246.

[21] E. Camponogara, D. Jia, B.H. Krogh, S.N. Talukdar, Distributed model predictive control, IEEE Control Systems Magazine 22 (1) (2002) 44-52.

[22] W.B. Dunbar, R.M. Murray, Model predictive control of coordinated multi-vehicle formations, in: Proceedings of the Conference on Decision and Control, Las Vegas, Nevada, 2002, pp. 4631-4636.

[23] S.Y. Li, Y. Zhang, Q.M. Zhu, Nash-optimization enhanced distributed model predictive control applied to the Shell benchmark problem, Information Sciences 170 (2-4) (2005) 329-349.

[24] Y. Zhang, S.Y. Li, Networked model predictive control based on neighbourhood optimization for serially connected large-scale processes, Journal of Process Control, 17 (1) (2007) 37-50.

[25] M. Mercangöza and F. J. Doyle, Distributed model predictive control of an experimental four-tank, Journal of Process Control, 17 (3) (2007) 297-308

[26] G.P. Liu, Y.Q. Xia, J. Chen, et al., Networked predictive control of systems with random network delays in both forward and feedback channels, IEEE Transactions on Industrial Electronics, 54 (3) (2007) $1282-1297$

[27] G.P. Liu, Y.Q. Xia, D. Rees and W.S. Hu, Design and stability criteria of networked predictive control systems with random network delay in the feedback channel, IEEE Transactions on Systems, Man, and Cybernetics, Part C: Applications and Reviews, 37 (2) (2007) 173-184

[28] E.I. Jury, Theory and application of Z-transform method, Wiley, New York, (1964)

[29] I. Izadi, Q. Zhao and T. Chen. An opimal scheme for fast rate fault detection based on multirate sampled data. Journal of Process Control, 15 (2005) 307-319 
[30] L. Hu, P. Shi and B. Huang. Stochastic stability and robust control for sampled-date systems with Markovian jump parameters. Journal of Mathematical Analysis and Applications, 313 (2006) 504-517

[31] I. Izadi, Q. Zhao and T. Chen. An b approach to fast rate fault detection for multirate sampled-data systems. Journal of process control, 16 (2006) 651-658

[32] H. Lin, G. Zhai and P.J. Antsaklis. Robust stability and attenuation analysis of a class of networked control systems. In: Proceedings of 42nd IEEE Conference on Decision and Control, Maui, Hawaii, USA, 2003, pp. 1182-1187

[33] D. Georgiev, D. M. Tilbury. Packet-based control: The H2-optimal solution. Automatica, 42 (2006) $137-144$

[34] E. Saber, An Introduction to Difference Equations, Springer, San Antonio, (2000)

[35] M. Maróti, B. Kusy, G. Simon, and Ákos Lédeczi. The flooding time synchronization protocol, in: Proceedings of the $2^{\text {nd }}$ international conference on Embedded networked sensor systems, 2004, pp. 39-49. 


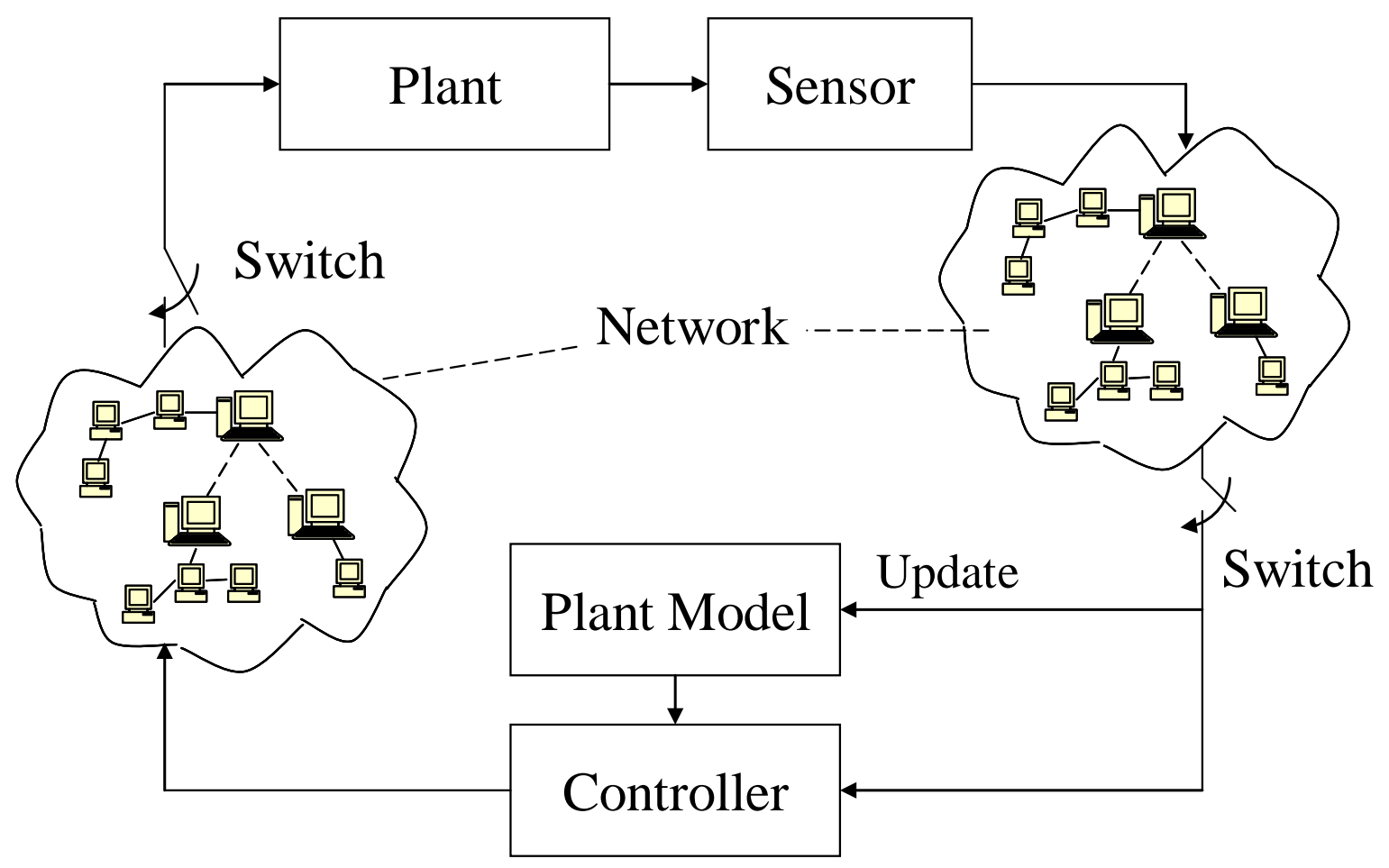

Fig. 1. Model based output feedback control for NCSs

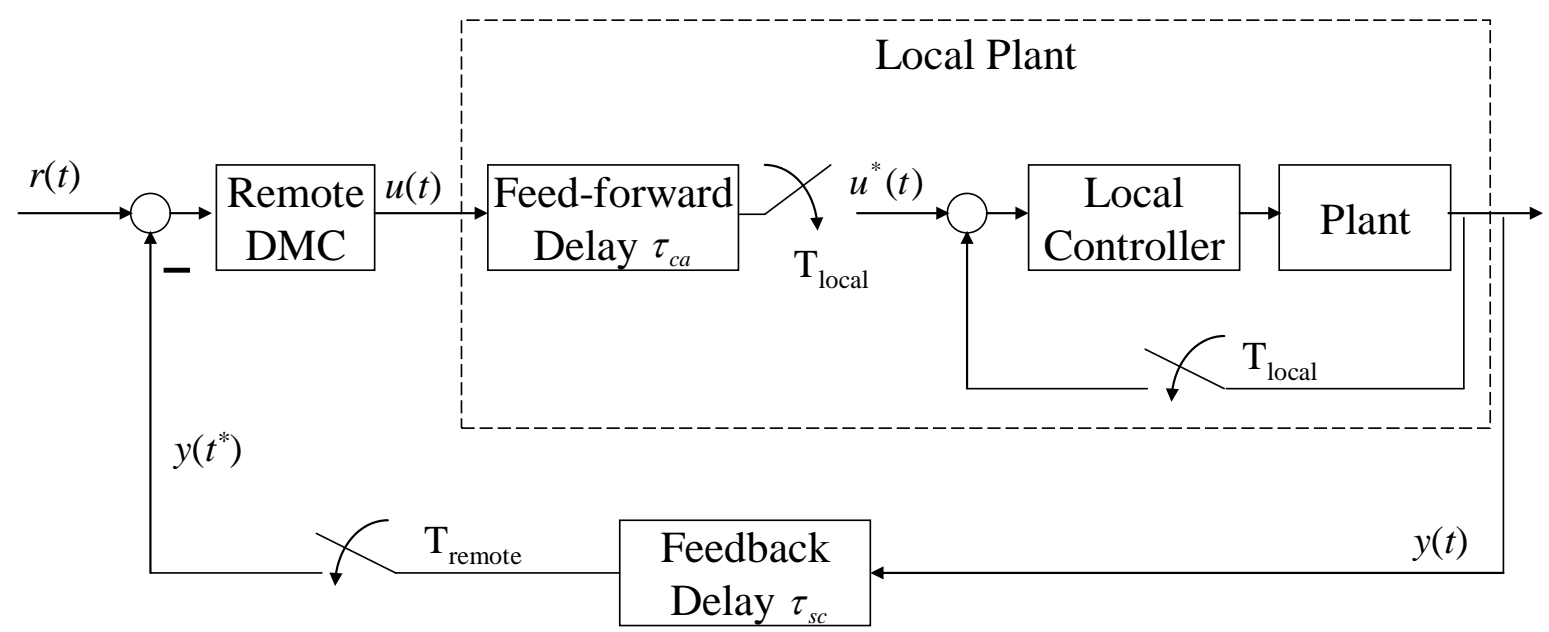

Fig. 2. Control structure with random access time in the sensor-controller links 


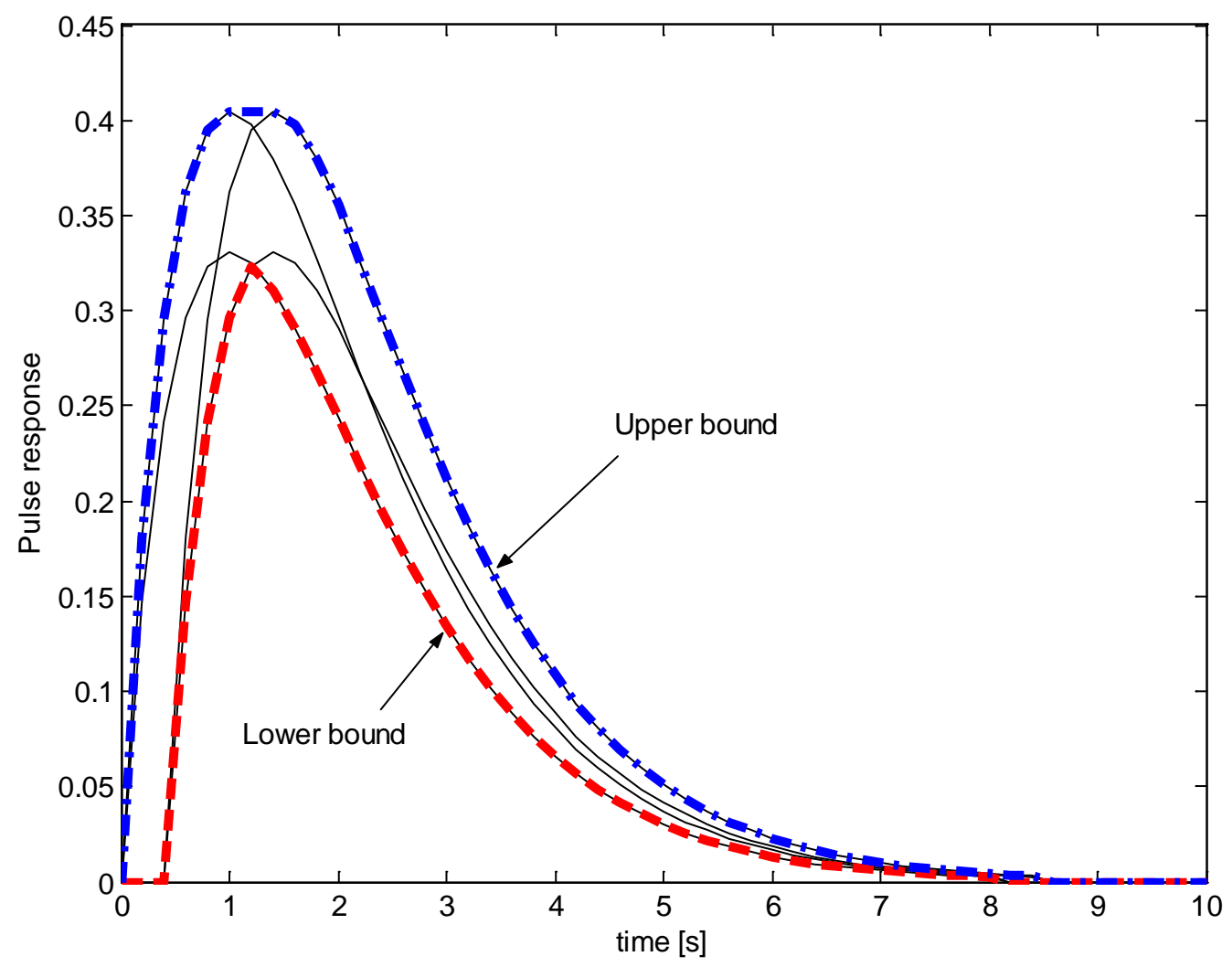

Fig. 3. A family of plants with an uncertain gain and dead time known to lie within the bounds [0.9, 1.1] and $[0,0.3]$ respectively 

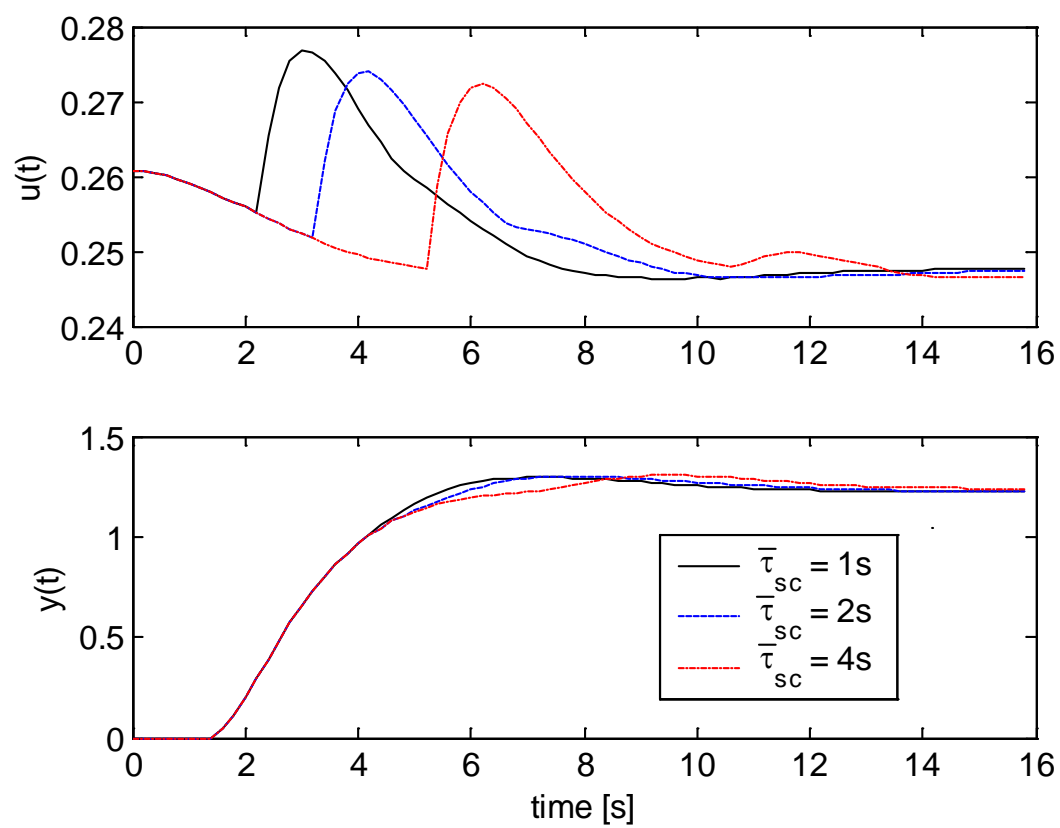

Fig. 4. The output trajectories of the remote DMC controller and the plant when using maximum delayed output feedback method
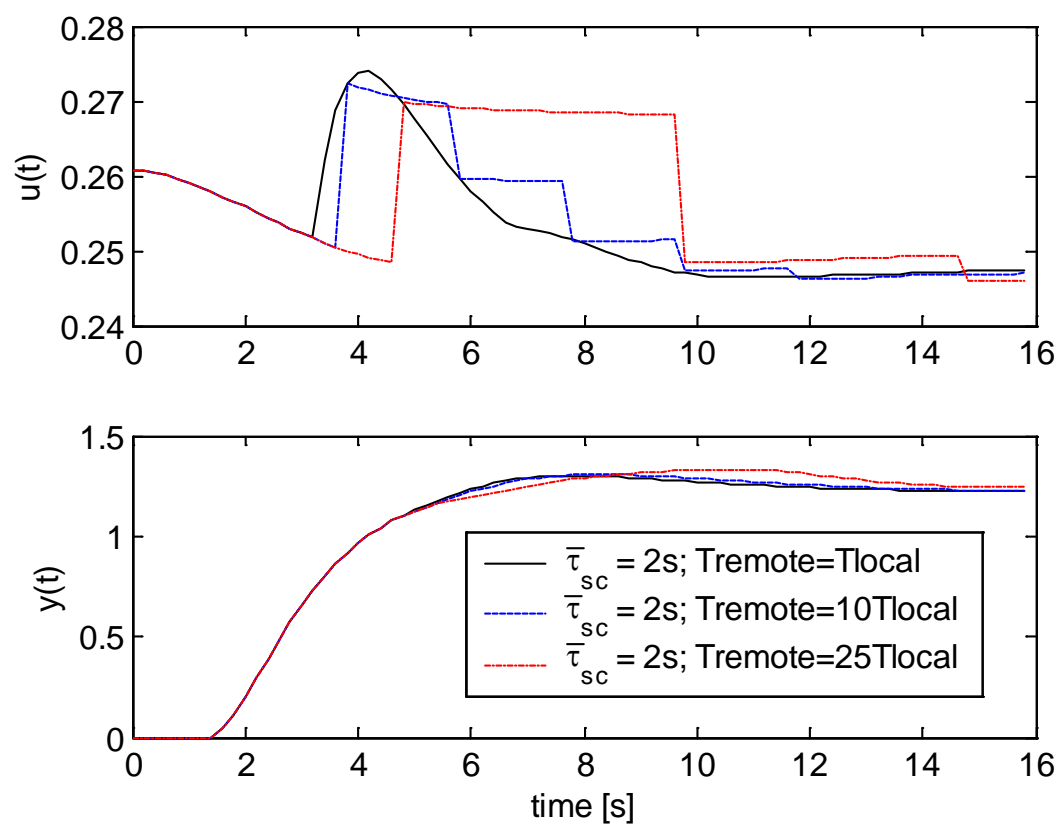

Fig. 5. The output trajectories of the remote DMC controller and the plant when using multi-rate sampling method 